\title{
Diabetes and COVID19: a bidirectional relationship
}

\author{
Ranjit Unnikrishnan (1D ${ }^{1 凶}$ and Anoop Misra ${ }^{2,3,4}$ \\ (c) The Author(s) 2021
}

The advent and rapid spread of the coronavirus disease-2019 (COVID19) pandemic across the world has focused attention on the relationship of commonly occurring comorbidities such as diabetes on the course and outcomes of this infection. While diabetes does not seem to be associated with an increased risk of COVID19 infection per se, it has been clearly demonstrated that the presence of hyperglycemia of any degree predisposes to worse outcomes, such as more severe respiratory involvement, ICU admissions, need for mechanical ventilation and mortality. Further, COVID19 infection has been associated with the development of new-onset hyperglycemia and diabetes, and worsening of glycemic control in pre-existing diabetes, due to direct pancreatic damage by the virus, body's stress response to infection (including cytokine storm) and use of diabetogenic drugs such as corticosteroids in the treatment of severe COVID19. In addition, public health measures taken to flatten the pandemic curve (such as lockdowns) can also adversely impact persons with diabetes by limiting their access to clinical care, healthy diet, and opportunities to exercise. Most antidiabetic medications can continue to be used in patients with mild COVID19 but switching over to insulin is preferred in severe disease.

European Journal of Clinical Nutrition (2021) 75:1332-1336; https://doi.org/10.1038/s41430-021-00961-y

\section{INTRODUCTION}

Coronavirus disease 2019 (COVID19), caused by the novel severe acute respiratory syndrome-coronavirus 2 (SARS-CoV2) is the most significant pandemic to have affected humanity in the last 100 years. As of March 2021, more than 120 million documented infections have occurred worldwide, with more than 2.6 million deaths. As the COVID19 pandemic spreads, it appears likely that a significant proportion of the world's population will be infected at some time or the other. Therefore, the interaction of COVID19 with other commonly occurring medical conditions needs to be studied so as to anticipate and thereby better manage the effects that they may have on one another.

Diabetes is one such comorbidity that affects more than 430 million people worldwide as of 2019 [1] and has the potential to unfavorably modify the natural history of COVID19. Conversely, COVID19 itself has been postulated to cause diabetes and to worsen glycemic control in pre-existing diabetes. Over the past year, a number of narrative, as well as systematic reviews analysing the link between diabetes and COVID19 infection, have been published [2-6], and their conclusions are summarized in Boxes 1 and 2 . In this article, we attempt to build upon these earlier studies and critically analyse the bidirectional link between these two conditions, providing insights into the clinical implications of the relationship wherever applicable.

\section{EFFECTS OF HYPERGLYCEMIA ON COVID19 Diabetes and risk of COVID}

Individuals with diabetes do not seem to be at higher risk of being infected with SARS-CoV2, compared to the general population
[2-4]. This is not entirely unexpected, as SARS-CoV2 generally affects the upper respiratory tract to begin with, and diabetes is in general not associated with a demonstrable increase in risk of upper respiratory tract infections (URTI) [5]. It is also possible that individuals with diabetes tend to follow non-pharmacological measures, such as mask wearing and social distancing more stringently than the general population, since they appreciate their higher risk of adverse outcomes of infection; this could have driven down infections in this population and masked a true increase in biological susceptibility.

Hyperglycemia and risk of adverse outcomes with COVID. In contrast to individuals without diabetes, COVID-19 infection in those with diabetes is associated with an increased risk of adverse outcomes such as more severe disease, pneumonia, ICU admissions, need for assisted ventilation and mortality. It is, however, not clear how much of this excess risk can be attributed to hyperglycemia, as people with diabetes (especially type 2 diabetes) have a higher frequency of other risk factors for severe COVID19 (such as advanced age, obesity, and cardiovascular disease) compared to those without diabetes.

A. Pre-existing diabetes and risk of adverse outcomes. It has been shown that among individuals with known diabetes, poor glycemic control preceding COVID19 infection (as measured by glycated hemoglobin levels) independently predicts adverse outcomes [6]. This reinforces the need for patients with all types of diabetes to maintain tight glycemic control during the pandemic period, a recommendation that might prove challenging to implement, given the difficulties in accessing chronic care,

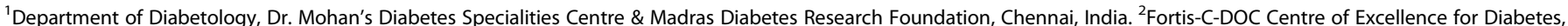

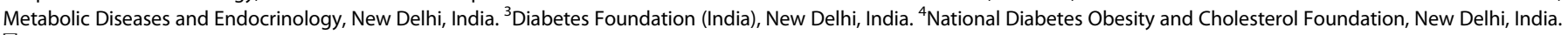

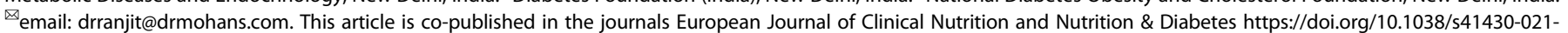
00961-y or https://doi.org/10.1038/s41387-021-00163-2

Received: 19 March 2021 Revised: 4 June 2021 Accepted: 8 June 2021 
Box 1. Pre-existing diabetes and COVID19: risks

- No increased risk of COVID19 infection

- Increased risk of adverse infection outcomes (severe disease, ICU admission, need for assisted ventilation and mortality), even at levels of hyperglycemia lower than those suggestive of diabetes

- Increased risk of "long COVID" (tiredness, breathlessness, muscle and joint pain inability to focus)

\footnotetext{
Box 2. COVID19 on diabetes: effects and use of antidiabetic medications

- Increased risk of development of new-onset hyperglycemia in infected individuals

- Worsening of glycemic control in pre-existing diabetes

- All classes of antidiabetic medications are suitable for use in mild cases of COVID19; insulin is to be preferred in severe cases
}

obtaining medications, and following diet and exercise guidelines during the pandemic and consequent lockdown [7]. The presence of diabetes mellitus was associated with a fourfold risk of adverse outcomes in a cohort of 339 patients from Wenzhou, China, even after adjusting for other potential confounders [8]. A clinic-registry based study from the United Kingdom showed that the presence of type 1 diabetes was associated with a 2.86 -fold increased risk and that of type 2 diabetes with a 1.8-fold increased risk of dying from COVID19 compared to individuals without diabetes [9]. This study also showed that the presence of diabetes complications such as cardiovascular disease and nephropathy was independently associated with risk of COVID mortality. Preliminary studies indicate that diabetes might also be associated with increased risk of long-term sequelae of COVID19 such as tiredness, muscle and joint pain, breathlessness, and inability to focus ("long COVID") [10].

B. Effect of mild degrees of hyperglycemia ("pre-diabetes") on COVID outcomes. There have been several studies looking at the effect of hyperglycemia (not necessarily in the range diagnostic of diabetes) on adverse COVID outcomes. Even slightly raised blood glucose levels (in individuals not hitherto known to have diabetes) have been shown to be associated with poor outcomes in COVID19. Wang et al. [11] studied 605 patients from two hospitals in Wuhan, China and found that those with fasting blood glucose (FBG) of 6.1 To $6.9 \mathrm{mmol} / \mathrm{l}(\mathrm{mg} / \mathrm{dL}$ here) at admission had a nearly threefold increased odds of 28-day inhospital complications, compared to those with FBG below $6.1 \mathrm{mmol} / \mathrm{I}$. From Guangdong (China), Zhang et al. [12] showed that FBG at admission accurately predicted 30-day adverse outcomes (acute respiratory distress syndrome, multiple organ dysfunction, ICU admissions, septic shock, or death), irrespective of the presence or absence of pre-existing diabetes. Indeed, it has recently been suggested that blood glucose levels should be considered as a "vital sign" while evaluating hospitalized patients with COVID19 infection [13].

C. Effect of new-onset hyperglycemia on COVID outcomes. In a study from Wuhan, it was found that the risk of ICU admission was highest for patients with new-onset diabetes, followed by individuals with known diabetes and was lowest in those with new-onset hyperglycemia without diabetes [14]. However, Bode et al. [15] have shown that hyperglycemia without diabetes is associated with worse outcome in COVID19 compared to preexisting diabetes. The poor outcomes associated with new-onset diabetes may reflect the fact that these hitherto undiagnosed and untreated patients would have more profound hyperglycemia and uncorrected metabolic risk factors compared to those with previously diagnosed diabetes [16]. The bulk of currently available evidence seems to suggest that new-onset hyperglycemia (irrespective of whether or not it meets the criteria for a diagnosis of diabetes) is associated with worse outcomes in COVID19 $[17,18]$.

Antidiabetic drug therapy in COVID19 infection. There have been no formal clinical trials comparing various antidiabetic agents in the context of COVID19 infection, so most of the available recommendations are based on expert opinion $[19,20]$. In general, there is no contraindication for the use of any of the available antidiabetic agents in the setting of mild to moderate COVID19 infection, and the patient can continue with his/ her usual drug regimen, provided glycemic control is appropriate [21]. In severe COVID19 infection, it is always advisable to switch the patient to insulin therapy [21].

\section{EFFECT OF COVID19 ON HYPERGLYCEMIA Hyperglycemia during COVID19 Infection}

There is now sufficient evidence to conclude that COVID19 infection is a diabetogenic state. Reports from China during the initial days of the pandemic suggested that COVID19 infection is associated with new-onset diabetes, often presenting as diabetic ketoacidosis (DKA) or hyperosmolar hyperglycemic state (HHS) and requiring exceedingly large doses of insulin for control of hyperglycemia [16, 22, 23]. A global registry has now been established to study the link between COVID19 and new-onset diabetes [24]. Misra et al. [25] have recently attempted to broadly classify new-onset hyperglycemia during the COVID pandemic into those with documented COVID19 infection and those without. Among individuals with documented COVID19 infection, more severe hyperglycemia was found in those with severe infection, probably due to a combination of factors such as cytokine storm, corticosteroid use, and direct beta-cell damage.

The diabetogenic effect of COVID19 may manifest not only as new-onset diabetes, but also as worsening of pre-existing diabetes. The mechanisms for the development of hyperglycemia in COVID19 can be broadly enlisted as follows (Fig. 1).

Direct virus-mediated beta-cell damage. The angiotensin converting enzyme (ACE) receptor, which acts as the portal of entry for SARS-CoV2, has been identified not only on respiratory epithelial cells, but also in the kidney, gastrointestinal tract, and the pancreas. SARS-CoV2 has been shown to infect and replicate in cells of the human endocrine and exocrine pancreas [26]. Entry of SARS-CoV2 into the beta cells of the pancreas, with subsequent cell destruction, has been postulated to underlie the development of new-onset, insulin-requiring diabetes in some patients with COVID19. The fact that a similar phenomenon has been described with SARS-CoV1 adds credence to this hypothesis [27]. It is generally agreed that $>90 \%$ of insulin-secreting cells need to be destroyed for this form of non-autoimmune diabetes to occur following a viral infection. In this respect, it is important to note that in contrast to SARS-Cov1, infection with SARS-CoV2 does seem to be associated with acute pancreatitis, with several cases having been reported in the literature $[28,29]$. Clinical course of such kind of hyperglycemia remains to be researched.

Triggering of beta-cell autoimmunity by virus. In addition to direct cytopathic effects, viruses have been postulated to cause diabetes by triggering autoimmune attack against pancreatic beta-cell antigens. Indeed, this is one of the most prevalent hypotheses related to the etiopathogenesis of type 1 diabetes, with Coxsackie $B$ virus, mumps virus, cytomegalovirus, rubella virus, and 


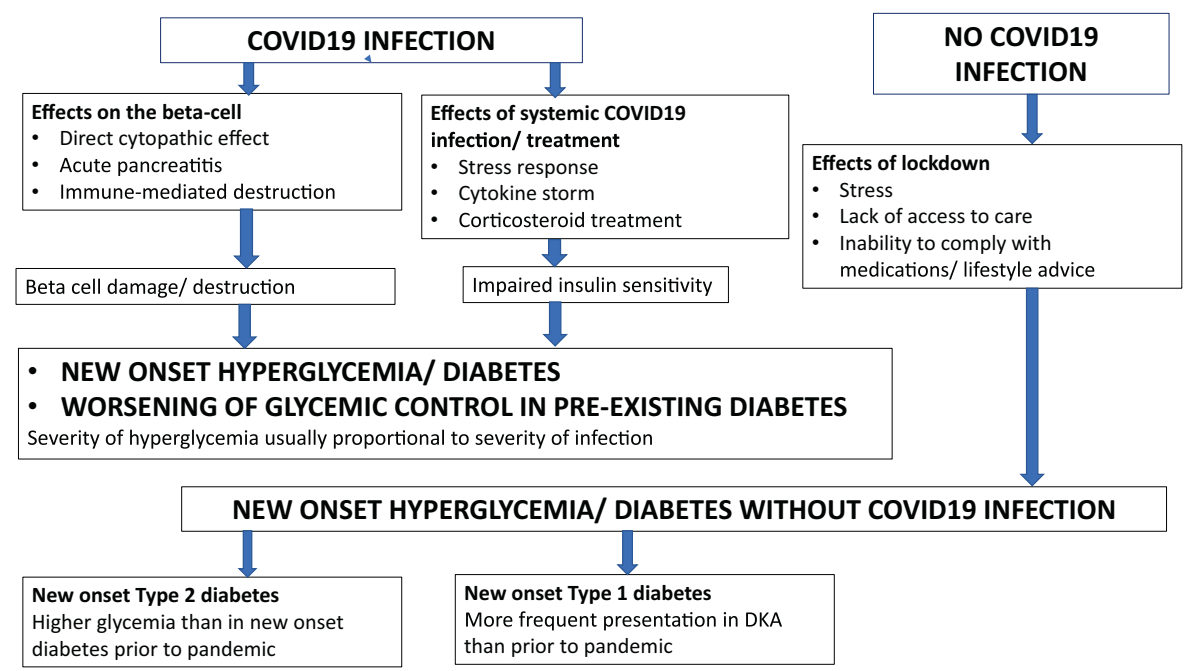

Fig. 1 Hyperglycemic states during COVID pandemic.

enteroviruses being the most commonly implicated agents. This theory holds that limited virus-mediated damage to beta cells releases hitherto sequestered antigens that lead to activation of autoreactive T-lymphocytes, culminating in an autoimmune response that ultimately destroys the remainder of the beta-cell mass, leading to insulin-dependent type 1 diabetes [30]. This process usually takes weeks to months and cannot explain the immediate onset of diabetes during the acute phase of COVID19 infection but may underlie disease development in some patients who develop diabetes in the weeks to months following recovery from infection. Research regarding this type of diabetes remains inadequate.

Diabetogenic effects of host responses to COVID19 infection. Host responses to COVID19 infection in the form of disorganized and exuberant immune response, can also lead to perturbations in glycemic status. As with any other acute infection, severe COVID19 is associated with non-specific activation of the immune system, with outpouring of counter-regulatory hormones and proinflammatory cytokines such as interleukin-6 (IL-6) and tumor necrosis factor (TNF) alpha, both of which are known to induce insulin resistance and hyperglycemia [31]. Sudden reduction in insulin sensitivity can precipitate diabetes in individuals with borderline beta-cell function and may even manifest as hyperglycemic crises in those with previously undiagnosed (and untreated) diabetes.

latrogenic hyperglycemia in COVID19. Following the publication of the interim findings of the Randomized Evaluation of Covid19 Therapy (RECOVERY) trial, corticosteroids such as dexamethasone have become the mainstay of management of severe COVID19 infection [32]. While these drugs are highly effective in preventing clinical deterioration and death in COVID19 pneumonia, they are also known to be highly diabetogenic drugs, and hyperglycemia is virtually inevitable when they are used at the doses prescribed for this indication. In individuals with undiagnosed diabetes or pre-diabetes, profound hyperglycemia often leading to DKA or HHS can occur. While the presence of hyperglycemia cannot be considered a contraindication to corticosteroid use in a lifethreatening situation such as COVID19 pneumonia, assessment of glycemic status is mandatory prior to using these drugs so that appropriate preventive/ therapeutic steps can be taken to minimize the magnitude of hyperglycemia.

\section{Hyperglycemia during COVID19 pandemic in individuals without COVID19 infection}

In this context, it should not be forgotten that the COVID19 pandemic and the consequent policy responses such as lockdowns, have had profound effects on the lives of individuals with pre-existing diabetes. Analysis of a large French database showed that COVID-19-induced lockdowns were associated with farreaching changes in dietary habits and physical activity levels, and these changes occurred in both favorable and unfavorable directions [33]. In many parts of the world, individuals with diabetes have faced difficulties in achieving requisite amounts of physical activity and accessing healthy foods during the pandemic [34]. Many of these patients would have put off their regular physician visits for fear of contracting the virus from the hospital or physician's office. Deterioration in glycemic control is therefore not an unexpected consequence of the COVID19 pandemic, and predictions have been published as to the magnitude of this deterioration [35]. However, it is heartening to note that a few studies have actually shown improvement of glycemic control during the COVID pandemic $[36,37]$. This may be due to increased awareness of the deleterious effects of diabetes particularly with reference to COVID, as well as patients having had more time to manage their diabetes in the "new normal" of working from home. There could, however, be a selection bias in these studies as only well-motivated patients would have ventured to seek clinic-based care during the lockdown.

Ghosh et al. [38] analyzed 282 patients with new-onset diabetes diagnosed during the COVID pandemic and compared them with 273 individuals newly diagnosed with diabetes immediately prior to the pandemic. They found that individuals with new-onset diabetes during the COVID pandemic had higher fasting and postprandial glucose levels and glycated hemoglobin compared to those diagnosed before the pandemic, even though the two groups did not differ significantly in any other respect including exposure to COVID (as measured by SARS-CoV2 antibody levels). Interestingly, based on data from multiple studies, Misra et al. [25] have stated that individuals without COVID19 infection who were diagnosed with either type 1 or type 2 diabetes during the pandemic, tended to present with more severe hyperglycemia than was the case prior to the pandemic (with a higher frequency of DKA in those presenting with type 1 diabetes). The worse metabolic profile of new-onset diabetes during the COVID pandemic is probably a reflection of increased stress, reduced physical activity and access to healthy foods and delayed diagnosis 
due to reluctance to visit physicians and hospitals during the pandemic and subsequent lockdown. It is likely that exacerbation in income inequalities following the lockdown will further worsen health outcomes, especially in countries like India where most healthcare is paid for "out-of-pocket" by the patient [39].

\section{CONCLUSIONS}

The bidirectional relationship between COVID19 and hyperglyce$\mathrm{mia} /$ diabetes presents a major challenge to healthcare systems as the pandemic spreads across the globe. It is essential that individuals with pre-existing diabetes get their blood glucose levels under control at the earliest so as to minimize adverse outcomes of COVID19, should they contract the infection. At the same time, physicians involved in the care of patients with COVID19 should be aware of the diabetogenic potential of this virus and look for new-onset hyperglycemia and diabetes in their patients, especially those treated with corticosteroids.

\section{REFERENCES}

1. International Diabetes Federation. IDF Diabetes Atlas $9^{\text {th }}$ Edition 2019. Available at https://www.diabetesatlas.org/en// [Accessed on 24 ${ }^{\text {th }}$ February 2021].

2. Apicella M, Campopiano MC, Mantuano M, Mazoni L, Coppelli A, Del Prato S. COVID-19 in people with diabetes: understanding the reasons for worse outcomes. Lancet Diabetes Endocrinol. 2020;8:782-92.

3. Li B, Yang J, Zhao F, Zhi L, Wang X, Liu L, et al. Prevalence and impact of cardiovascular metabolic diseases on COVID-19 in China. Clin Res Cardiol. 2020;109:531-8.

4. Fadini GP, Morieri ML, Longato E, Avogaro A. Prevalence and impact of diabetes among people infected with SARS-CoV-2. J. Endocrinol. Invest. 2020;43:867-9.

5. Unnikrishnan R, Misra A. Infections and diabetes: risks and mitigation with reference to India. Diabetes Metab. Syndr. 2020;14:1889-94.

6. Williamson G, Guidinger C, Kelly NR. Factors associated with COVID-19-related hospital death in the linked electronic health records of 17 million adult NHS patients. J Chem Inf Model. 2019;53:1689-99.

7. Ghosh A, Arora B, Gupta R, Anoop S, Misra A. Effects of nationwide lockdown during COVID-19 epidemic on lifestyle and other medical issues of patients with type 2 diabetes in north India. Diabetes Metab. Syndr. 2020;14:917-20.

8. Targher G, Mantovani A, Wang XB, Yan HD, Sun QF, Pan KH, et al. Patients with diabetes are at higher risk for severe illness from COVID-19. Diabetes Metab. 2020;46:335-7.

9. Barron E, Bakhai C, Kar P, Weaver A, Bradley D, Ismail H, et al. Associations of type 1 and type 2 diabetes with COVID-19-related mortality in England: a wholepopulation study. Lancet Diabetes Endocrinol. 2020;8:813-22.

10. Feldman EL, Savelieff MG, Hayek SS, Pennathur S, Kretzler M, Pop-Busui R. COVID-19 and diabetes: a collision and collusion of two diseases. Diabetes. 2020;69:2549-65.

11. Wang $S, M a P$, Zhang $S$, Song $S$, Wang $Z$, Ma $Y$, et al. Fasting blood glucose at admission is an independent predictor for 28-day mortality in patients with COVID-19 without previous diagnosis of diabetes: a multi-centre retrospective study. Diabetologia. 2020;63:2102-11.

12. Zhang B, Liu S, Zhang L, Dong Y, Zhang S. Admission fasting blood glucose predicts 30-day poor outcome in patients hospitalized for COVID-19 pneumonia. Diabetes Obes. Metab. 2020;22:1955-7.

13. Kesavadev J, Misra A, Saboo B, Aravind SR, Hussain A, Czupryniak L, et al. Blood glucose levels should be considered as a new vital sign indicative of prognosis during hospitalization. Diabetes Metab. Syndr. 2021;15:221-7.

14. Zhang Y, Li H, Zhang J, Cao Y, Zhao X, Yu N, et al. The clinical characteristics and outcomes of patients with diabetes and secondary hyperglycaemia with coronavirus disease 2019: A single-centre, retrospective, observational study in Wuhan. Diabetes Obes. Metab. 2020;22:1443-54.

15. Bode B, Garrett V, Messler J, McFarland R, Crowe J, Booth R, et al. Glycemic characteristics and clinical outcomes of COVID-19 patients hospitalized in the United States. J. Diabetes Sci. Technol. 2020;14:813-21.

16. Li H, Tian S, Chen T, Cui Z, Shi N, Zhong X, et al. Newly diagnosed diabetes is associated with a higher risk of mortality than known diabetes in hospitalized patients with COVID-19. Diabetes Obes. Metab. 2020;22:1897-906.

17. Singh AK, Singh R. Hyperglycemia without diabetes and new-onset diabetes are both associated with poorer outcomes in COVID-19. Diabetes Res Clin Pract. 2020;167:108382.

18. Stefan N, Birkenfeld AL, Schulze MB, Ludwig DS. Obesity and impaired metabolic health in patients with COVID-19. Nat. Rev. Endocrinol. 2020;16:341-2.
19. Bornstein SR, Rubino F, Khunti K, Mingrone G, Hopkins D, Birkenfeld AL, et al. Practical recommendations for the management of diabetes in patients with COVID-19. Lancet Diabetes Endocrinol. 2020;8:546-50.

20. Gupta R, Ghosh A, Singh AK, Misra A. Clinical considerations for patients with diabetes in times of COVID-19 epidemic. Diabetes Metab. Syndr. 2020;14:211-2.

21. Singh AK, Gupta R, Ghosh A, Misra A. Diabetes in COVID-19: Prevalence, pathophysiology, prognosis and practical considerations. Diabetes Metab. Syndr. 2020;14:303-10

22. Chee YJ, Ng SJH, Yeoh E. Diabetic ketoacidosis precipitated by Covid-19 in a patient with newly diagnosed diabetes mellitus. Diabetes Res Clin. Pract. 2020;164:108166.

23. Ren $H$, Yang $Y$, Wang $F$, Yan $Y$, Shi $X$, Dong $K$, et al. Association of the insulin resistance marker TyG index with the severity and mortality of COVID-19. Cardiovasc Diabetol. 2020;19:58.

24. Rubino F, Amiel SA, Zimmet P, Alberti G, Bornstein S, Eckel RH, et al. New-onset diabetes in Covid-19. N Engl J Med. 2020;383:789-90.

25. Misra A, Ghosh A, Gupta R. Heterogeneity in presentation of hyperglycaemia during COVID-19 pandemic: a proposed classification. Diabetes Metab. Syndr. 2021;15:403-6.

26. Müller JA, Groß R, Conzelmann C, Krüger J, Merle U, Steinhart J, et al. SARS-CoV-2 infects and replicates in cells of the human endocrine and exocrine pancreas. Nat. Metab. 2021;3:149-65.

27. Yang JK, Lin SS, Ji XJ, Guo LM. Binding of SARS coronavirus to its receptor damages islets and causes acute diabetes. Acta Diabetol. 2010;47:193-9.

28. Wang F, Wang $H$, Fan J, Zhang $Y$, Wang $H$, Zhao Q. Pancreatic injury patterns in patients with coronavirus disease 19 pneumonia. Gastroenterology. 2020;159:367-70.

29. de-Madaria E, Capurso G. COVID-19 and acute pancreatitis: examining the causality. Nat. Rev. Gastroenterol. Hepatol. 2021;18:3-4.

30. Boddu SK, Aurangabadkar G, Kuchay MS. New onset diabetes, type 1 diabetes and COVID-19. Diabetes Metab. Syndr. 2020;14:2211-7.

31. Papachristou S, Stamatiou I, Stoian AP, Papanas N. New-Onset Diabetes in COVID19: Time to Frame Its Fearful Symmetry. Diabetes Ther. 2021;12:461-4.

32. RECOVERY Collaborative Group, Horby P, Lim WS, Emberson JR, Mafham M, Bell $\mathrm{JL}$ et al. Dexamethasone in hospitalized patients with covid-19-preliminary report. N Engl J Med. 2020;384: 693-704.

33. Deschasaux-Tanguy $M$, Druesne-Pecollo $N$, Esseddik $Y$, de Edelenyi FS, Allès $B$, Andreeva VA. et al. Diet and physical activity during the coronavirus disease 2019 (COVID-19) lockdown (March-May 2020): results from the French NutriNet-Santé cohort study. Am J Clin Nutr. 2021;113:924-38.

34. Jayawardena R, Misra A. Balanced diet is a major casualty in COVID-19. Diabetes Metab. Syndr. 2020;14:1085-6.

35. Ghosal S, Sinha B, Majumder M, Misra A. Estimation of effects of nationwide lockdown for containing coronavirus infection on worsening of glycosylated haemoglobin and increase in diabetes-related complications: A simulation model using multivariate regression analysis. Diabetes Metab. Syndr. 2020;14:319-23.

36. Anjana RM, Pradeepa R, Deepa M, Jebarani S, Venkatesan U, Parvathi SJ, et al. Acceptability and utilization of newer technologies and effects on glycemic control in type 2 diabetes: lessons learned from lockdown. Diabetes Technol. Ther. 2020;22:527-34.

37. Fernández E, Cortazar A, Bellido V. Impact of COVID-19 lockdown on glycemic control in patients with type 1 diabetes. Diabetes Res Clin. Pract. 2020;166:108348.

38. Ghosh A, Anjana RM, Shanthi Rani CS, Jeba Rani S, Gupta R, Jha A, et al. Glycemic parameters in patients with new-onset diabetes during COVID-19 pandemic are more severe than in patients with new-onset diabetes before the pandemic: NOD COVID India Study. Diabetes Metab. Syndr. 2020;15:215-20.

39. Raman R, Rajalakshmi R, Surya J, Ramakrishnan R, Sivaprasad S, Conroy D, et al. Impact on health and provision of healthcare services during the COVID-19 lockdown in India: a multicentre cross-sectional study. BMJ Open. 2021;11:e043590.

\section{COMPETING INTERESTS}

The authors declare no competing interests.

\section{ADDITIONAL INFORMATION}

Correspondence and requests for materials should be addressed to R.U.

Reprints and permission information is available at http://www.nature.com/ reprints

Publisher's note Springer Nature remains neutral with regard to jurisdictional claims in published maps and institutional affiliations. 
1336

(c) Open Access This article is licensed under a Creative Commons Attribution 4.0 International License, which permits use, sharing, adaptation, distribution and reproduction in any medium or format, as long as you give appropriate credit to the original author(s) and the source, provide a link to the Creative Commons license, and indicate if changes were made. The images or other third party material in this article are included in the article's Creative Commons license, unless indicated otherwise in a credit line to the material. If material is not included in the article's Creative Commons license and your intended use is not permitted by statutory regulation or exceeds the permitted use, you will need to obtain permission directly from the copyright holder. To view a copy of this license, visit http://creativecommons. org/licenses/by/4.0/.

(c) The Author(s) 2021 